\title{
DISPROOF OF SOME CONJECTURES OF K.RAMACHANDRA
}

\author{
BY \\ JOHAN ANDERSSON
}

\section{INTRODUCTION}

In a recent paper [9] K.Ramachandra states some conjectures, and gives consequences in the theory of the Riemann zeta function. In this paper I will will present two different disproofs of them. The first will be an elementary application of the Szasz-Müntz theorem. The second will depend on a version of the Voronin universality theorem, and is also slightly stronger in the sense that it disprove a weaker conjecture. An elementary(but more complicated) disproof has been given by Rusza-Lazkovich [11].

\section{DISPROOF OF SOME CONJECTURES}

2.1 The conjectures. I will first state the three conjectures as given by Ramachandra [9], and Ramachandra-Balasubramanian [5].

Conjecture 1. For all $N \geq H \geq 1000$ and all $N$-tuples $a_{1}=1, a_{2}, \ldots, a_{N}$ of complex numbers we have

$$
\frac{1}{H} \int_{0}^{H}\left|\sum_{n=1}^{N} a_{n} n^{i t}\right| d t \geq 10^{-1000}
$$

Conjecture 2. For all $N \geq H \geq 1000$ and all $N$-tuples $a_{1}=1, a_{2}, \ldots, a_{N}$ of complex numbers we have when $M=H(\log H)^{-2}$

$$
\frac{1}{H} \int_{0}^{H}\left|\sum_{n=1}^{N} a_{n} n^{i t}\right|^{2} d t \geq(\log H)^{-1000} \sum_{n=1}^{M}\left|a_{n}\right|^{2}
$$

\footnotetext{
${ }^{*}$ Recieved on 07-09-1999.

1991 Mathematics Subject Classfication. Primary 11M41, Secondary 42A65, 11 N30.

Key words and phrases. Dirichlet series, Titchmarsh series.
} 
Conjecture 3. There exist a constant $c>0$ such that

$$
\int_{0}^{T}\left|\sum_{n=1}^{N} a_{n} n^{i t}\right|^{2} d t \geq c \sum_{n \leq c T}\left|a_{n}\right|^{2}
$$

2.2 The Szász-Müntz theorem To disprove these conjectures, we first consider the following classic result of Szász

Lemma 1. (Szász) If we have that

$$
\sum_{n=1}^{\infty} \frac{1+2 \operatorname{Re}\left(\lambda_{n}\right)}{1+\left|\lambda_{n}\right|^{2}}=+\infty
$$

where $\Re\left(\lambda_{n}\right) \geq 0$ then the set of finite linear combinations of $x^{\lambda_{n}}$ is dense in $L^{2}(0,1)$

Proof See Szász [12], theorem A.

I will now state a theorem that will effectively disprove the above conjectures:

Theorem 1. For each $D \geq 0$ and $\varepsilon>0$ there exists an $N \geq 0$ and complex numbers $a_{2}, \ldots, a_{N}$, such that

$$
\int_{0}^{D}\left|1+\sum_{n=2}^{N} a_{n} n^{i t}\right|^{2} d t \leq \varepsilon
$$

Proof Since $-1 \in L^{2}(0,1)$ and

$$
\sum_{n=2}^{\infty} \frac{1+2 \Re(-i \log n)}{1+|-i \log n|^{2}}=+\infty
$$

we have by the lemma that for each $\delta>0$ there exists an $N>0$ and complex numbers $a_{2}, \ldots, a_{N}$ such that

$$
\int_{0}^{1}\left|1+\sum_{n=2}^{N} a_{n} x^{-i \log n}\right|^{2} d x<\delta
$$

We obtain

$$
\begin{gathered}
\delta>\int_{0}^{1}\left|1+\sum_{n=2}^{N} a_{n} x^{-i \log n}\right|^{2} d x \geq \int_{e^{-D}}^{1}\left|1+\sum_{n=2}^{N} a_{n} x^{-i \log n}\right|^{2} d x= \\
=\text { Substituting } t=-\log x)=
\end{gathered}
$$




$$
\int_{0}^{D} e^{-t}\left|1+\sum_{n=2}^{N} a_{n} n^{i t}\right|^{2} d t \geq e^{-D} \int_{0}^{D}\left|1+\sum_{n=2}^{N} a_{n} n^{i t}\right|^{2} d t
$$

By choosing $\delta=e^{-D} \varepsilon$ we obtain the theorem.

It is now an easy task to falsify the conjectures.

Proposition. Conjectures 1, 2 and 3 are false.

Proof. For conjecture 1 , choose $\varepsilon<10^{-2000} H$ and $D=H$ in Theorem 1 and apply the Cauchy-Schwarz inequality

$$
\int_{0}^{H}\left|\sum_{n=1}^{N} a_{n} n^{i t}\right| d t \leq \sqrt{H} \sqrt{\int_{0}^{H}\left|\sum_{n=1}^{N} a_{n} n^{i t}\right|^{2} d t}
$$

For conjecture 2, choose $\varepsilon<(\log H)^{-1000} H$, and $D=H$ in Theorem. To disprove Conjecture 3, chose e.g. $a_{1}=T=1$, and $\epsilon=c / 2$.

2.3. The Voronin Theorem. In private correspondence, Ramachandra asked whether the conjectures hold under the additional growth assumption $\left|a_{k}\right| \ll(H k)^{100}$. Ramachandra and Balasubramanian have proved conjectures 1 and 2 under the this and the additional further assumption that $N<\exp (\exp (c H))$. This shows that $N$ must be very large compared to $H$ for the conjectures to be false. However, they are in fact still false, although their proof requires a deeper result. A version of the Voronin universality theorem for the Riemann zeta-function. We will state the theorem that shows that the conjectures are still false below:

Theorem 2. Suppose that $H, \varepsilon>0,0<\delta<\frac{1}{2}$. Then there exists $\left|a_{k}\right| \leq k^{\delta-1}$ such that

$$
\max _{t \in[0, H]}\left|1+\sum_{n=2}^{N} a_{n} n^{i t}\right| d t<\varepsilon
$$

The idea goes as follows: We use the following version of the Voronin universality theorem

Theorem 3. (Voronin-Bagchi) For any compact subset $K$ of the complex numbers such that $x \in K \Longrightarrow \frac{1}{2}<\operatorname{Re}(x)<1$, non vanishing analytic function $f$ on $K$ and $\varepsilon>0$, we have a real $t$ such that $|\zeta(z+i t)-f(z)| \leq \epsilon$, for all $z \in K$ 
Proof. See Bagchi [1].

and the following version of the approximate functional equation for the Riemann zetafunction

$$
\left|\zeta(\sigma+i t)-\sum_{k=1}^{N} k^{-\sigma-i t}\right| \leq C N^{\sigma-1} \text {, for e.g. } t<N<2 t, \sigma \geq \frac{1}{2}
$$

Proof (of Theorem 2) Choose $T_{0}$ so that when $T>T_{0}$ then

$$
\left|\zeta(1-\delta+i t)-\sum_{k<2 T} k^{\delta-1-i t}\right|<\frac{\varepsilon}{3}
$$

when $T \leq t \leq T+H$. Now chose $T>T_{0}$ so that

$$
|\zeta(1-\delta+i t)|<\frac{\varepsilon}{3}
$$

for $T \leq t \leq T+H$ (This is possible by applying Theorem 3 to $f(z)=\varepsilon / 3$, and $K=$ $[1-\delta, 1-\delta+i H])$. Using the triangle inequality we get

$$
\left|\sum_{k<2 T} k^{\delta-1-i t}\right|<\varepsilon
$$

for all $T<t<T+H$. Now we can chose $N=T$ and $a_{n}=n^{\delta-1-i T}$ in the theorem.

\section{Summary}

In [8] K. Ramachandra stated a similar conjecture to conjectures 1 and 2. Although it was more general in the sense that it considered Dirichlet series of form $A(s)=1+\sum a_{n} \lambda_{n}^{-s}$, for certain $\lambda_{n}$ generalizing $\lambda_{n}=n$ it was much weaker as it had three further conditions. $H$ depended on $N,\left|a_{n}\right|$ were bounded from above and $A$ was bounded in certain regions in the complex plane. Under these additional assumptions, $A(s)$ is called a Titchmarshseries and for these, analogues of conjectures 1 and 2, and similar conjectures were proved in Ramachandra [7], and Balasubramanian and Ramachandra [2], [4] and [3]. It would certainly be interesting to see if all these additional assumptions are needed, or if a certain subset of 
them implies the truth of conjectures 1 and 2. For a disproof of the $L^{p}(0, D), p>2$ version of conjectures 1 and 2, or for considering much more sparse sequences than $\log n$ in (1), we need a stronger version of the Lemma, the reader is referred to the literature on the theory of completeness of complex exponentials, see e.g. the classic by Levinson [6] or Redheffer [10] for a more recent survey. It should be noticed that problems similar to conjectures 1 and 2 are also studied in Turán power sum theory (although there it is essentially the $\lambda_{n}$ 's which vary, instead of the $a_{n}$ 's), see Turán [13] for a thorough treatment.

\section{R E F E R E N C S}

1.Bhaskar Bagchi, A joint universality theorem for Dirichlet l-functions. (English), Math. Z. 181 (1982), no. 3, 319-334.

2. R.Balasubramanian and K.Ramachandra, Proof of some conjectures on the mean-value of Titchmarsh series. I, Hardy-Ramanujan J.13 (1990), 1-20.

3._-_, Proof of some conjectures on the mean-value of Titchmarsh series. II HardyRamanujan J. 14 (1991), 1-20.

4.-_- Proof of some conjectures on the mean-value of Titchmarsh series. III, Proc. Indian Acad. Sci. Math. Sci. 102(1992), no.2, 83-91.

5. —_- On Riemann zeta-function and allied questions.II, Hardy-Ramanujan J. 18 (1995), 10-22.

6. Norman Levinson, Gap and Density Theorems, American Mathematical Society, New York, 1940, American Mathematical Society Colloquium Publications, v. 26.

7. K.Ramachandra, Progress towards a conjecture on the mean-value of Titchmarsh series1, Recent progress in Analytic number theory (H.Halberstam and C.Hooley, eds.), vol.1, Academic Press, London, New York, Toronto, Sydney, San Fransisco, 1981, pp. 303-318. 
8. K.Ramachandra, Proof of some conjectures on the mean-value of Titchmarsh series with applications to Titchmarsh's phenomenon, Hardy-Ramanujan J. 13 (1990), 21-27.

9 —, On Riemann zeta-function and allied questions,Astérisque (1992), no.209, 57-72, Journées Arithmétiques, 1991 (Geneva).

10. Raymond M. Redheffer, Completeness of sets of complex exponentials, Advances in Math. 24 (1977), no.1, 1-62.

11. M.Rusza, I.Lazkovich, Sums of periodic functions and a problem of Ramachandra, Tech. report, Mathematical institute of the Hungarian Academy of Sciences, 1996.

12. 0.Szász, Über die Approximation stetiger Funktionendurch lineare Aggregate von Potenzen, Math Ann. 77 (1916),482-496.

13. Paul Turán, On a new method of analysis and its applications, Pure and Applied Mathematics, John Wiley \& Sons Inc., New York, 1984, With the assistance of G. Halász and J. Pintz, With a foreword by Vera T.Sós, A Wiley-Interscience Publication.

Address of the Author:

Prof.Johan Andersson

Department of Mathematics

Stockholm University

S-10691, Stockholm

SWEDEN.

E-mail:- johana@matematik.su.se 